\title{
VEGF-D-enhanced lymph node metastasis of ovarian cancer is reversed by vesicular stomatitis virus matrix protein
}

\author{
XIAORONG QI ${ }^{1 *}$, LICHENG DU $^{2 *}$, XIANCHENG CHEN $^{3}$, LIJUAN CHEN $^{3}$, TAO YI $^{1}$, \\ XIANG $\mathrm{CHEN}^{3}$, YANJUN WEN ${ }^{3}$, YUQUAN WEI ${ }^{3}$ and XIAZHAO ${ }^{1}$ \\ ${ }^{1}$ Department of Gynecology and Obstetrics, Key Laboratory of Obstetric and Gynecologic and Pediatric Diseases \\ and Birth Defects of Ministry of Education, West China Second Hospital, State Key Laboratory of Biotherapy, \\ Sichuan University, Chengdu, Sichuan 610041; ${ }^{2}$ Provincial Hospital Affiliated to Shandong University, Jinan; \\ ${ }^{3}$ State Key Laboratory of Biotherapy/Collaborative Innovation Center of Biotherapy, \\ West China Hospital, Sichuan University, Chengdu, Sichuan 610041, P.R. China
}

Received January 3, 2016; Accepted April 25, 2016

DOI: $10.3892 /$ ijo.2016.3527

\begin{abstract}
Lymphatic metastasis is a poor prognostic factor in ovarian cancer, which correlates to the majority of cancer deaths. Matrix protein (MP) of vesicular stomatitis virus (VSV) exhibits potent antitumor and antiangiogenic activities through inducing apoptosis and inhibiting angiogenesis. In this study, the antitumor and antimetastatic effects of MP were further investigated. Wild-type SKOV3 (WT-SK) cells were successfully transfected with empty vector pcDNA3.1 plasmid, or pcDNA3.1-VEGF-D recombinant plasmid to construct cell lines named EV-SK, and VEGFD-SK, respectively. Inhibition of VEGFD-SK cell migration and invasion was detected by Transwell and wound healing assay. Then, lymphogenous metastatic model of ovarian cancer was established by injecting VEGFD-SK cells subcutaneously into the left hindlimb claw pad of nude mice. The inducted apoptotic effect of MP on VEGFD-SK cells were assessed by flow analysis and Hoechst-33258 staining, respectively, in vitro. The in vivo antitumor and antiangiogenic activities of MP gene were evaluated with lymphogenous metastatic model of ovarian cancer. Tumor volume and lymphatic metastasis
\end{abstract}

Correspondence to: Dr Xia Zhao, Department of Gynecology and Obstetrics, Key Laboratory of Obstetric and Gynecologic and Pediatric Diseases and Birth Defects of Ministry of Education, West China Second Hospital, State Key Laboratory of Biotherapy, Sichuan University, Chengdu, Sichuan 610041, P.R. China

E-mail:xia-zhao@163.com

${ }^{*}$ Contributed equally

Abbreviations: VEGF-D, vascular endothelial growth factor D; VSV, vesicular stomatitis virus; MP, matrix protein; LN, lymph node; RT-PCR, reverse transcription-polymerase chain reaction; MMP-2, matrix metalloproteinase-2, LYVE-1, human lymphatic vessel endothelial hyaluronan receptor-1

Key words: VEGF-D, lymph node metastasis, vesicular stomatitis virus matrix protein, ovarian cancer rates were measured. Lymphatic vessels were delineated using Evan's blue and LYVE-1 staining. Expression of VEGF-D and MMP-2 were evaluated by immunostaining. Apoptosis of tumor cells was analyzed by Hoechst-33258 staining. Mice bearing VEGFD-SK tumor cells displayed more rapid tumorigenesis, higher lymphogenous metastatic tendency and increased lymphatic vessel density compared with the mice bearing WT-SK or EV-SK cells. However, VEGF-D-enhanced metastasis was evidently reversed by MP. MP significantly reduced the invasion of VEGFD-SK cells, tumor volume, lymphatic metastasis rates and lymphatic vessel density compared with control groups $(\mathrm{P}<0.05)$, accompanied with down-expression of VEGF-D and MMP-2 and increased apoptosis. Our data indicate that MP has strong antitumor and antimetastatic abilities, and it may be a promising therapeutic strategy against the lymphatic metastasis of human ovarian cancer.

\section{Introduction}

Ovarian cancer is the deadliest malignancy of the female reproductive tract (1). The 5-year survival rate is $>90 \%$ when the disease is limited to the ovary; however, it decreases to only $30 \%$ when the disease has extended (2). Lymphatic metastasis is one of the prognostic parameters for ovarian cancer and may account for a majority of deaths (3-5). Patients with negative lymph nodes (LNs) survived significantly longer than those who had positive lymph nodes (6). Despite advances in surgical management and chemotherapy, lymphatic metastasis remains an obstacle in cancer therapy. Accordingly, control of lymph node metastasis is an important strategy for the treatment of ovarian cancer.

The lymph-angiogenic growth factor, vascular endothelial growth factor-D (VEGF-D) is involved in the growth of lymphatic vessels and promotion of lymph node metastasis $(7,8)$. VEGF-D stimulates angiogenesis and lymphangiogenesis as well as accelerates tumor growth. It is essential for the development of lymphatic vessels within tumors, and therefore promotes metastatic spread of tumor cells via the lymphatic 
route $(9,10)$. In this study, we established a feasible high lymphogenous metastatic model using SKOV3 cells transfected with pcDNA3.1-VEGF-D plasmid and then introduced it to experimental cancer therapy.

Vesicular stomatitis virus (VSV), a negative-stranded RNA rhabdovirus with a single genome that encodes five proteins (nucleoprotein $\mathrm{N}$, phosphoprotein $\mathrm{P}$, matrixprotein M, glycoprotein $\mathrm{G}$, and polymerase $\mathrm{L}$ ), can preferentially replicate in many types of tumor cells. Recent studies have demonstrated that matrix protein (MP) of VSV is capable of inducing apoptosis in the absence of other viral components in vitro mainly through inhibiting host gene expression at the level of transcription (11-14) and nucleocytoplasmic transport of host RNA and proteins $(15,16)$. In addition, MP could cause inactivation of Akt, resulting in dominant inhibition of Akt/ protein kinase B signaling (17). Therefore, MP possesses potent antitumor and anti-angiogenesis properties in various tumors (18-20). It has also been observed abolishing ascites formation via inhibiting VEGF production (21). However, it is still unclear whether MP could effectively inhibit lymphangiogenesis and node metastasis.

In this study, we tested the antitumor and antimetastasis efficacy of a recombinant plasmid DNA carrying MP-cDNA in the lymphogenous metastatic model. Cationic liposomes were used as the gene delivery system. Our data demonstrated that VEGFD-enhanced lymphatic metastasis and lymphangiogenesis were reversed by MP. The expression of VEGF-D and matrix metalloproteinase-2 (MMP-2) were inhibited when treated with pVAX-MP liposome complex. Suppression of tumor growth and increase of apoptosis were also detected. MP may become a promising therapeutic strategy against lymph node metastasis of ovarian cancer without systemic toxic effects.

\section{Materials and methods}

Cell lines. Human epithelial serous cystadenocarcinoma cell line SKOV3 cells were obtained from the American Type Culture Collection (ATCC; Rockville, MD, USA), and cultured in RPMI-1640 medium (Gibco) supplemented with $10 \%$ fetal calf serum, $100 \mathrm{U} / \mathrm{ml}$ penicillin, and $100 \mathrm{mg} / \mathrm{ml}$ streptomycin. Cells were maintained in a $37^{\circ} \mathrm{C}$ humidified incubator with $5 \%$ $\mathrm{CO}_{2}$ atmosphere.

Establishment of VEGF-D overexpressing clonal line. VEGF-D cDNA was cloned from mouse ovarian cDNA library (Stratagene, La Jolla, CA, USA). The pcDNA3.1/VEGF-D was a gift from Dr Xinjiang Xie. The SKOV3 cells were transfected with either the recombinant pcDNA3.1/VEGF-D or pcDNA3.1 (empty vector) as control. The transfected cells were cultured in G418 selection medium (400 $\mu \mathrm{g} / \mathrm{ml})$ for screening. G418resistant colonies which carried VEGF-D plasmid stably were re-useable for additional studies. Wild-type SKOV3 cells, SKOV3 cells transfected with pcDNA3.1 plasmid and SKOV3 cells transfected with recombinant pcDNA3.1/VEGF-D plasmid were named as WT-SK, EV-SK, and VEGFD-SK cells, respectively.

$R T-P C R$. The total RNA was extracted by TRIzol reagent (Invitrogen, Carlsbad, CA, USA) according to the manufacturer's instructions. Expression of MP in vitro was confirmed with RT-PCR. The upstream and downstream primers for MP (GenBank accession no. EU917223.1) were: 5'-CGAAC GACCTACACCGAAC-3', and 5'-CCCTTCAGACCGAGA ATCTT-3', respectively. Expression of VEGF-D in vitro and in vivo were detected by RT-PCR. The primer sequences of VEGF-D were as follow: 5'-GCAAGCTTATGTATGGAGAA TGGGGAATG-3', and 5'-CGTCTAGATCAAGGGTTCTC CTGGCTG-3' (amplifies nucleotides 1,077 bp of mouse VEGF-D, Genbank accession no. GI6753873) (22). GAPDH was used as an internal control.

Preparations of plasmid liposome complexes. pVAX plasmid (Invitrogen, San Diego, CA, USA) encoded MP was constructed as previously described (23). pVAX without MP was used as control. The large-scale plasmid DNA was prepared using Endofree Plasmid Giga kit (Qiagen, Chatsworth, CA, USA). Cationic liposomes were gifts from Dr Chen and Professor Chen (State Key Laboratory of Biotherapy) (24). Plasmids and cationic liposomes were diluted in RPMI-1640 medium without serum or D5W (dextrose 5\% in water), and left at room temperature for 30 min. The pVAX liposome complexes were named pVAX:lip. The pVAX-MP liposome complexes were named pVAX-MP:lip.

Cell transfection and flow analysis. VEGFD-SK cells $\left(2 \times 10^{5} /\right.$ well) were seeded in 6-well dishes and incubated to $80 \%$ confluence. pVAX:lip (containing $2 \mu \mathrm{g} / \mathrm{ml}$ pVA plasmid) or pVAX-MP:lip (containing $2 \mu \mathrm{g} / \mathrm{ml}$ pVAX-MP plasmid) was prepared in $2 \mathrm{ml}$ RPMI-1640 medium without serum. VEGFD-SK cells were incubated with the complexes for $6 \mathrm{~h}$. Then, VEGFD-SK cells were supplemented with medium containing serum for further $48 \mathrm{~h}$. Untreated VEGFD-SK cells were needed as control. Quantitative evaluation of apoptotic VEGFD-SK cells was performed by flow cytometric analysis after Annexin $\mathrm{V}$ binding and propidium iodide staining (Invitrogen).

Transwell assay. Invasion inhibition was quantified with a Transwell invasions assay. The cells were harvested, seeded and transfected with plasmid complex for $48 \mathrm{~h}$ according to the above method. Matrigel invasion chambers (Millipore, $8 \mu \mathrm{m}$ ) were hydrated with serum-free media. The ovarian cancer cells were stained and counted by trypan blue. The cells $\left(4 \times 10^{4}\right)$ were resuspended with serum-free medium in the top of the chamber, and $72 \mathrm{~h}$ later fixed by with $4 \%$ neutral formalin. The chambers were stained with $0.1 \%$ crystal violet. The cells at the top of the Matrigel membrane were removed through washing in $\mathrm{dH}_{2} \mathrm{O}$. The number of the invading cells on the lower surface was assessed by an inverted microscope (x100).

Wound healing assay. Monolayer wound healing assay was used to assess the role of pVAX-MP:lip in cell migration. The cells were transfected in 24-well culture plates for $48 \mathrm{~h}$. Then, the cells were detached, resuspended and counted. The equal number of cells were cultured in each well of a 6-well culture dish. Twenty-four hours later, the monolayer was confluent. Parallel scratch wounds with similar widths were created on the monolayer with $100 \mu \mathrm{m}$ micropipette tip. Migration of cells into the wound was observed using phase contrast microscopy (x100). Cells that migrated into the wounded area or cells with extended protrusions from the border of the wound were 
photographed every $6 \mathrm{~h}$. The status of wound closure was evaluated by inverted microscope.

Tumor models. Athymic nude mice (BALB/c, 6-8-week-old, non-fertile, 18-20 g) were purchased from Sichuan University Animal Center, and were maintained in pathogen-free conditions with sterile chow. The nude mice were randomly assigned to three groups (six mice /group). WT-SK, EV-SK, VEGFD-SK cells were injected subcutaneously with $2 \times 10^{6}$ cells into the left hind paws of mice respectively (25). The tumor volume was determined by the following formula: tumor volume $\left(\mathrm{mm}^{3}\right)=0.52 \mathrm{x}$ length $(\mathrm{mm}) \mathrm{x}$ width $(\mathrm{mm}) \mathrm{x}$ height $(\mathrm{mm})$. All the animal experiments were approved by the Institute's Animal Care and Use Committee.

Therapy for VEGF-D-enhanced metastasis. Seven days following VEGFD-SK cells inoculation, the tumors in the left hind paws of mice were visible. Systemic therapy was initiated. The tumor-bearing mice were injected i.v. with the corresponding reagent at 5-day intervals for a total of five times (six mice/group): i) pVAX:lip group: mice were treated with $50 \mu \mathrm{g} \mathrm{pVAX} / 150 \mu \mathrm{g}$ liposome complexes (volume $=200 \mu \mathrm{l}$ ); ii) pVAX-MP:lip group: mice received $50 \mu \mathrm{g}$ pVAX-MP/150 $\mu \mathrm{g}$ liposome complexes.

Evan's blue visualization of lymph vessels. When tumors in foot grew to $\sim 1 \mathrm{~cm}$ in diameter, Evan's blue $(5 \mathrm{mg} / \mathrm{ml})$ was infused subcutaneously into the tumor tissue areas until lymph vessel (LV) draining to the popliteal lymph nodes were marked, and lymphatic network images were obtained.

Metastasis rates. The volume of local tumors, number and the location of metastasis in the lymph nodes were assessed. Specimens were fixed in $10 \%$ formalin for examination of hematoxylin and eosin (H\&E). Metastasis rates in the three barrier LNs, including popliteal space, parailiac, and renal hilum LNs in the drainage routes from footpad tumors were assessed, and metastasis rate was expressed as a ratio of metastatic lymph nodes counts out of the total number of lymph nodes in each barrier.

Immunohistochemistry. Deparaffinized sections were immersed in $0.01 \mathrm{M}$ citrate buffer and heated in an autoclave. Endogenous peroxidase activity was quenched in $3 \%$ hydrogen peroxide. Non-specific binding sites were blocked with homeotypic non-immunoglobulin of the secondary antibody at $37^{\circ} \mathrm{C}$. VEGF-D antibody (Boster Biomart, CN, USA) was used at a dilution of 1:100. MMP-2 antibody (Santa Cruz Biotechnology, CA, USA) was used as primary antibody at a dilution of 1:100. Sections were incubated with the primary antibody at $4^{\circ} \mathrm{C}$ overnight, biotinylated secondary antibody at $37^{\circ} \mathrm{C}$, and streptavidin-biotin-peroxidase complex (SABC) sequentially. The immunoreactions were visualized with diaminobenzidine (DAB) solution and the crimson yellow precipitates were identified as positive staining. Counterstaining was performed with hematoxylin.

Estimation of VEGF-D and MMP-2 staining. The levels of VEGF-D and MMP-2 staining were classified into four grades: $\pm(<25 \%$ of cells), meaning absent or very weak staining;
$+(25-50 \%$ of cells $)$, weak staining; $++(50-75 \%$ of cells $)$, moderate staining; and $+++(>75 \%$ of cells), strong staining. Faint or equivocal immunoreactions were scored as negative.

Computer-assisted morphometric analysis. Human lymphatic vessel endothelial hyaluronan receptor-1 (LYVE-1) antibody (Santa Cruz) was performed to identify lymphatic microvessels at local tumor tissues. Lymphatic microvessel counting was performed at high-power field (HPF) (x400), and measured using computer assisted morphometric analysis. Five high-power fields with abundant lymphatic sprout and highest lymphatic vessel density (LVD) were identified and captured by Spot digital camera. Parameters were evaluated using IP-Lab computer aided image analysis software.

Apoptosis analysis. Qualitative apoptosis was determined by Hoechst-33258 compounds (Sigma) according to the manufacturer's instructions. Condensed chromatin or fragmented nuclei emitting intense blue fluorescence were classified as apoptotic cells.

Toxicology analysis. The relevant indexes such as weight loss, skin, behavior and feeding were evaluated. The weight of mice was measured every four days. When the mice were sacrificed, tissues and organs (including lung, brain, liver, kidney, heart, pancreas and spleen) were fixed. The sections were evaluated with H\&E.

Statistical analysis. Statistical calculations were carried out using StatView statistical software version 8.2 (SAS Institute, Cary, N.C., USA). The $\chi^{2}$ test was used to analyze the lymphatic metastasis rates. Differences in tumor volumes, LVD and apoptosis index were assessed with analysis of variance (ANOVA). All results were expressed as means \pm standard deviation (SD). $\mathrm{P}<0.05$ was considered statistically significant.

\section{Results}

Expression of MP in vitro. RT-PCR analysis was employed to verify whether transfection of pVAX-MP:lip could result in an evidently improved expression level of MP in ovarian cancer cells. High expression of MP was detected in cells transfected with pVAX-MP:lip. GAPDH served as the internal positive control (Fig. 1A).

Expression of VEGF-D in vitro and in vivo. VEGF-D expression in vitro was evaluated using RT-PCR (Fig. 1B). Overexpression of VEGF-D mRNA was recognized in VEGFD-SK cells, but not in WT-SK and EV-SK cells. However, the overexpression of VEGF-D was inhibited in pVAX-MP:lip-treated VEGFD-SK cells. This expression of VEGF-D did not decrease obviously when VEGFD-SK cells were treated with pVAX:lip. VEGF-D expression in vivo was detected by RT-PCR and immunohistochemistry (Fig. 1C and D). As expected, overexpression of VEGF-D mRNA was confirmed in VEGFD-SK group. The expression of VEGF-D was suppressed when mice were treated with pVAX-MP:lip. However, the expression of VEGF-D was still obvious in the pVAX:lip group. VEGF-D protein in vivo was also evaluated with immunohistochemistry. Our data showed only very weak staining $( \pm)$ for VEGF-D protein in 

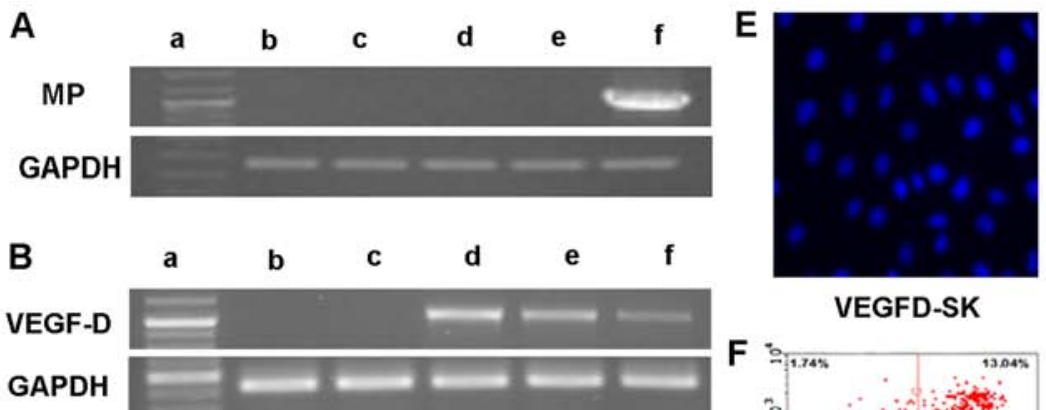

VEGFD-SK

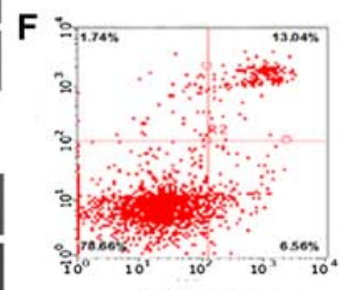

VEGFD-SK

C

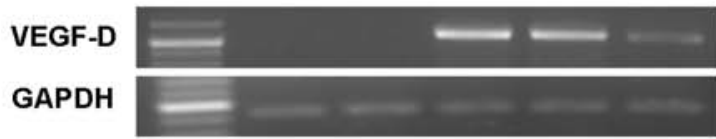

D

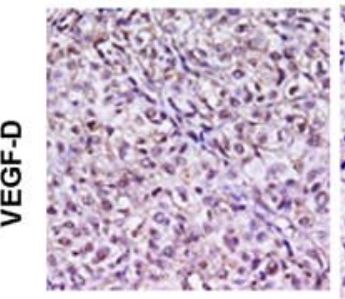

WT-SK

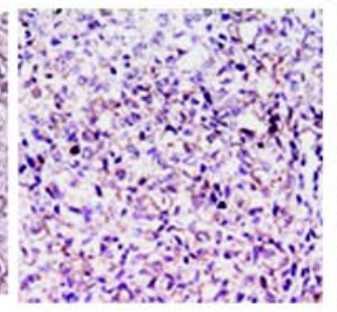

EV-SK

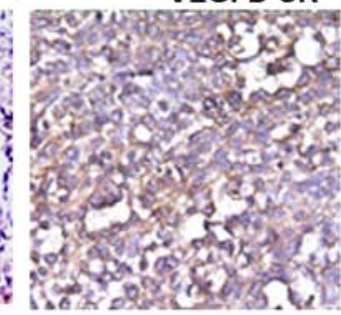

VEGFD-SK

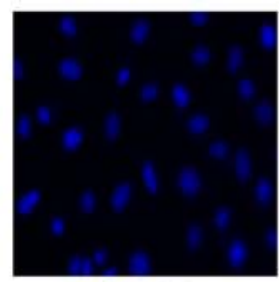

pVAX:lip
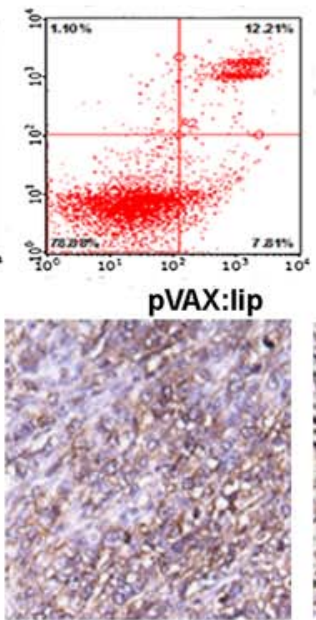

pVAX:lip

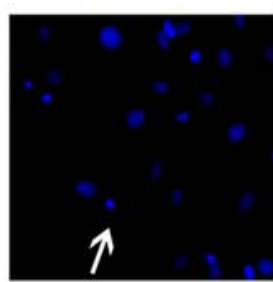

pVAX-MP:lip
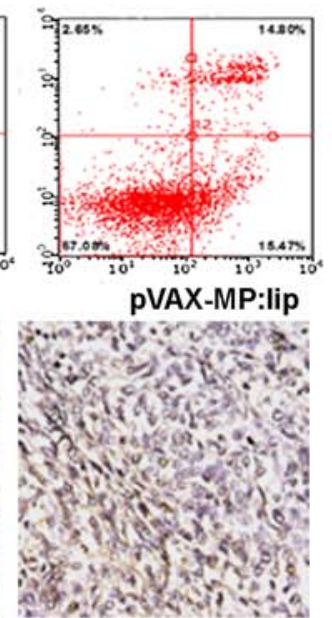

pVAX-MP:lip

Figure 1. RT-PCR and apoptotic effect on VEGFD-SK cells. (A) RT-PCR analysis of expression of MP in vitro. High expression of MP in VEGFD-SK cells transfected with pVAX-MP:lip was detected (1,036 bp). GAPDH was used for the internal standard. The displaying panel of electrophoretic image presents the following: DNA ladder marker (a), WT-SK (b), EV-SK (c), VEGFD-SK (d), pVAX:lip (e), and pVAX-MP:lip (f), respectively. (B) Overexpression of VEGF-D using RT-PCR could be detected in VEGFD-SK cells, but not in WT-SK and EV-SK cells (1,077 bp). However, the VEGF-D expression was suppressed when VEGFD-SK cells suffered from pVAX-MP:lip. The overexpression of VEGF-D did not decreased obviously when VEGFD-SK cells were treated with pVA. (C) VEGF-D expression in vivo was analysis by RT-PCR. (D) The expression of VEGF-D in vivo was determined with immunohistochemistry using VEGF-D antibody: VEGFD-SK tumors were detected with much stronger staining. Whereas, the VEGFD-SK tumors in pVAX-MP:lip group present nearly negative staining. Tumors in pVAX:lip group also exhibited strong staining. Very weak VEGF-D protein was observed in WT-SK and EV-SK tumors. (E) Hoechst-33258 stained fluorescence microscopy (x200) in vitro: apoptosis cells were observed apparently when the VEGFD-SK cells were treated with pVAX-MP:lip (arrow). (F) A quantitative comparison of apoptotic cells was carried out by flow analysis stained with Annexin V-FITC and propidium iodide: pVAX-MP significantly increased VEGFD-SK cells apoptosis compared with the controls.

WT-SK and EV-SK tumor tissues (Fig. 1D); however, tumors in VEGFD-SK group showed much stronger staining (+++) compared with tumors in the WT-SK or EV-ST groups, which indicated the overexpression of VEGF-D in VEGFD-SK tumors. Whereas, nearly negative staining was observed in tumors of pVAX-MP:lip group. Local tumors also exhibited VEGF-D staining obviously in pVAX:lip group.

Apoptosis of VEGFD-SK cells in vitro. pVAX-MP:lip-treated VEGFD-SK cells resulted in morphological changes characterized as apoptosis using Hoechst-33258 staining (Fig. 1E): a brightly fluorescent condensed nuclei and apoptotic bodies. However, these changes rarely occurred in pVAX:lip-treated or untreated VEGFD-SK cells. A quantitative comparison of apoptotic VEGFD-SK cells in vitro was carried out to evaluate the action of pVAX-MP using flow-cytometric analysis (Fig. 1F). Treatment with pVAX-MP significantly increased the populations of Annexin V-positive cells (apoptotic cells) in the right quadrants of flow cytometry graphs compared with empty vector and untreated group.

Inhibition of cell invasion and migration induced by $p V A X$ $M P$ :lip in vitro. Wound healing assay was used to analyze the migration of the cells. The cells in the VEGFD-SK group were nearly closed, demonstrating increased migration in VEGFD-SK group. However, the wound widths in the pVAXMP:lip group was wider than that in VEGFD-SK or pVAX:lip group, indicationg the significant effect of MP on cell migration (Fig. 2A). VEGFD-SK cells had much high migration ability, but pVAX-MP:lip group had the lowest migration tendency in wound healing assay (Fig. 2C) $(\mathrm{P}<0.05$ WT-SK group versus VEGFD-SK group; $\mathrm{P}<0.05$ pVAX-lip group versus pVAX:lip group).

The monolayer Transwell assays were performed to explore the role of MP in VEGFD-SK cell invasion. Consistent with the results of the wound healing assay, the number of invading cells in the VEGFD-SK group was increased compared with WT-SK or EV-SK group. While the number of invading cells in the pVAX-MP:lip group was clearly lower than that in the pVAX:lip group (Fig. 2B).

Tumor growth. Tumor volume was measured consecutively. As shown in Fig. 3, VEGFD-SK group has a faster growth rate of tumor compared with WT-SK, EV-SK groups. The tumor in VEGFD-SK group exhibited fast tumor formation and growth, similar to the tumor progression in pVAX:lip group. 
A
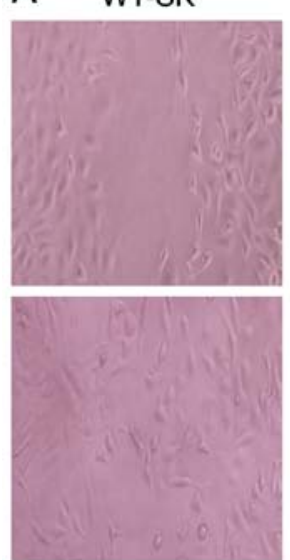

B

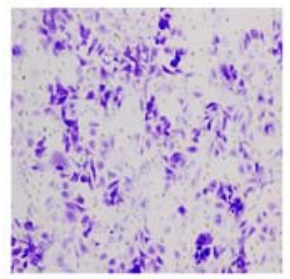

EV-SK
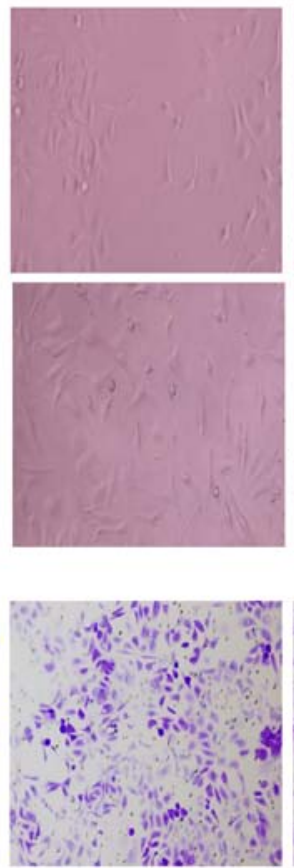

VEGFD-SK
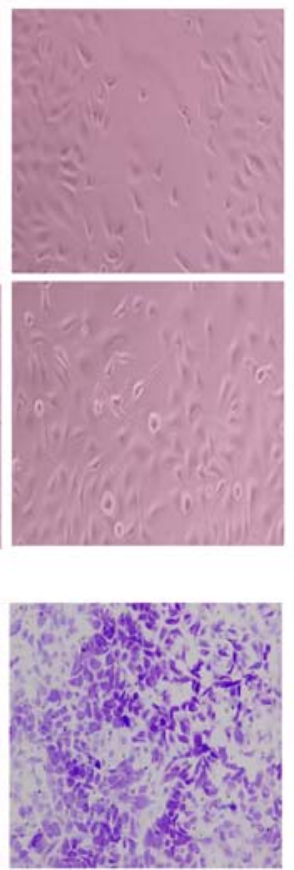

pVAX:lip
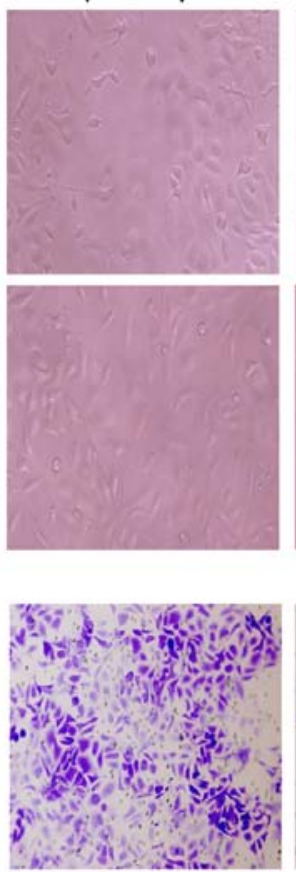

pVAX-MP:lip
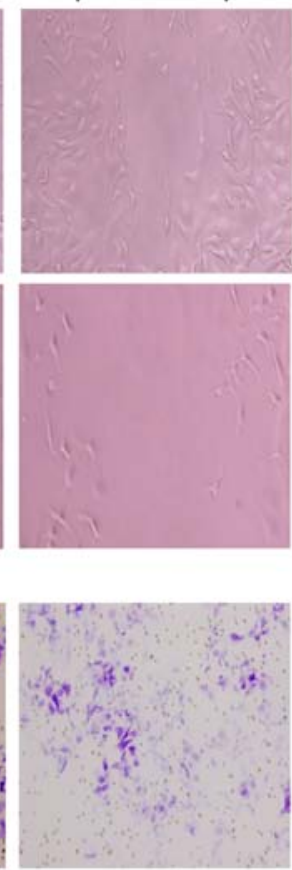

C

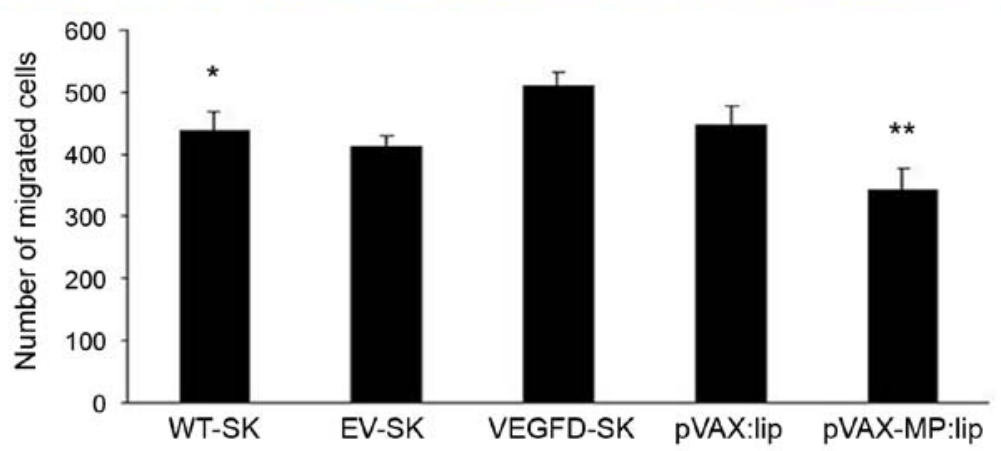

Figure 2. Inhibition of cell invasion and migration induced by pVAX-MP:lip in vitro. (A) Wound healing analysis of migration. The cell motility was enhanced in the VEGFD-SK group. However, the cell motility was decreased in the pVAX-MP:lip group: the wound widths in the pVAX-MP:lip group was wider than in VEGFD-SK or pVAX:lip group. (B) Transwell assay was performed to evaluate cell invasion. Migratory capacity was increased in VEGFD-SK cells (x100). The number of invading cells in the VEGFD-SK group was increased compared with other groups. While the numbers of invading cells in the pVAX-MP:lip groups were obviously lower than in the pVAX:lip group (x100). (C) VEGFD-SK cells had much high migration ability, but pVAX-MP:lip group had the lowest migration tendency in the wound healing assay ( $\left(\mathrm{P}<0.05\right.$ WT-SK group versus VEGFD-SK group; ${ }^{* *} \mathrm{P}<0.05 \mathrm{pVAX}$-lip group versus pVAX:lip group).

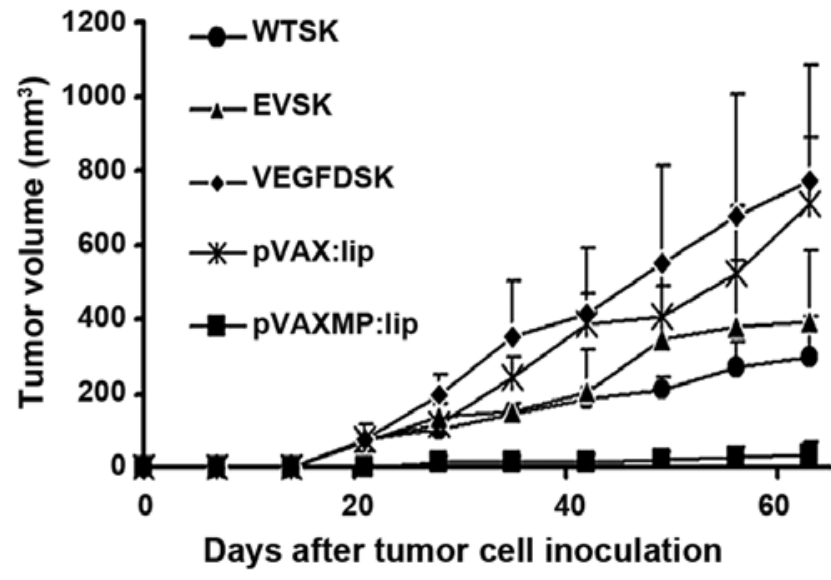

Figure 3. Tumor growth in nude mice. The graphs indicate that tumor volume in VEGFD-SK group was larger than it in WT-SK and EV-SK groups. However, the tumor growth in pVAXMP:lip group is extremely suppressed after systemic treatment $(\mathrm{P}<0.05$ versus any other group). The pVAX:lip group also exhibited faster tumor growth.
However, tumorigenesis and growth in pVAX-MP:lip group was extremely suppressed after treatment with pVAX-MP:lip ( $\mathrm{P}<0.05$ versus any other group).

Evan's blue in vivo visualization and microscopic lymphatic vessel density. Lymphangiogenesis were evaluated with lymphangiogram stained by Evan's blue (Fig. 4A, top panel) as well as LVD stained by LYVE-1 (Fig. 4A, middle panel). The staining revealed few LVs draining Evan's blue detected in the WT-SK and EV-SK groups. Twisted peritumoral blue LVs with dilated sprout clumps emerged around tumors in VEGFD-SK and pVAX:lip groups (arrow, blue lymphatic vessels), but no visible LVs were seen in pVAX-MP:lip group. Anti-LYVE-1 immunostaining showed that there were some LVs in WT-SK and EV-SK groups, especially sparse LVs in pVAX-MP:lip group. However, rich nascent lymphatic microvessels with dilated lumina were present in VEGFD-SK and pVAX:lip group. The tumor tissues in VEGFD-SK $(107.6 \pm 20.23)$ and pVAX:lip (103.4 \pm 17.86$)$ groups displayed overt enhancement 
A
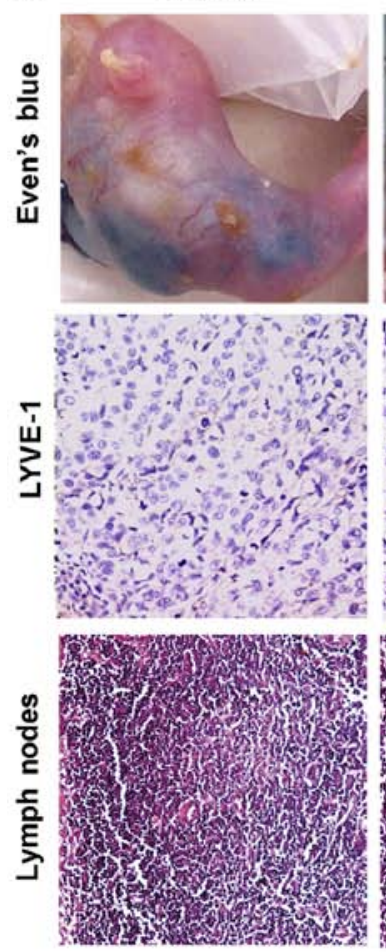

B

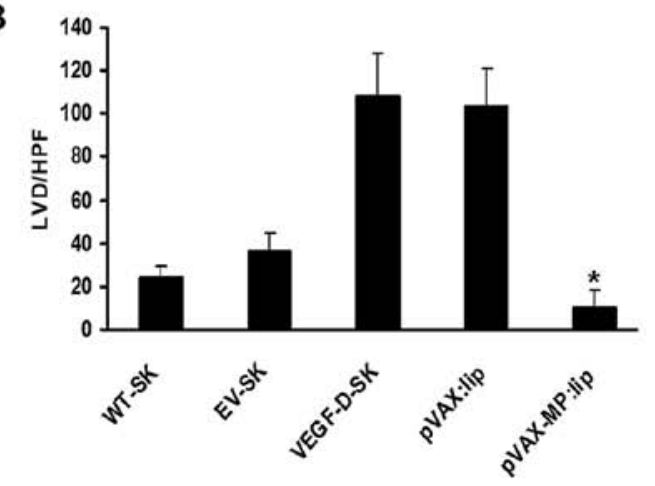

EV-SK
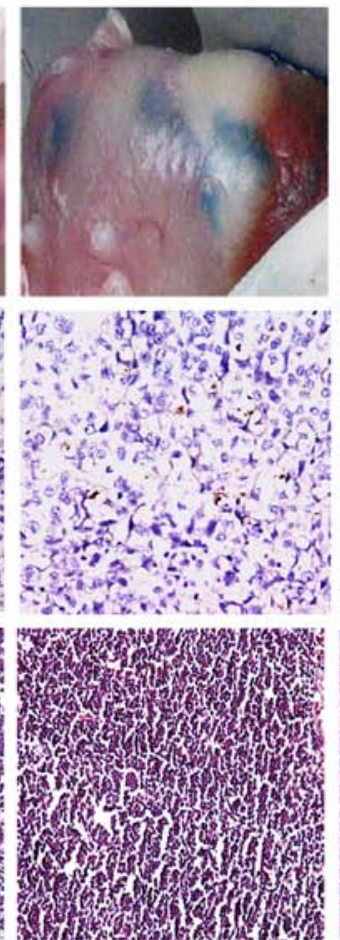

VEGFD-SK
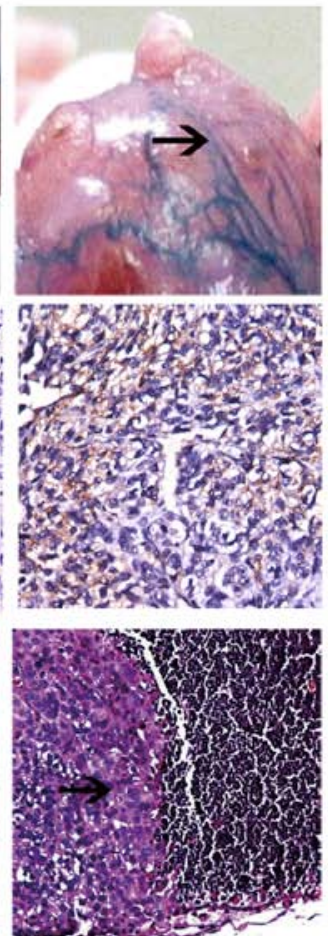

pVAX:lip
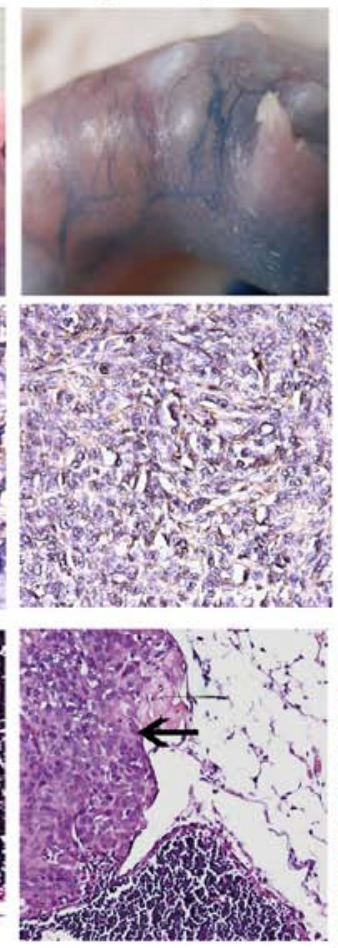

pVAX-MP:lip
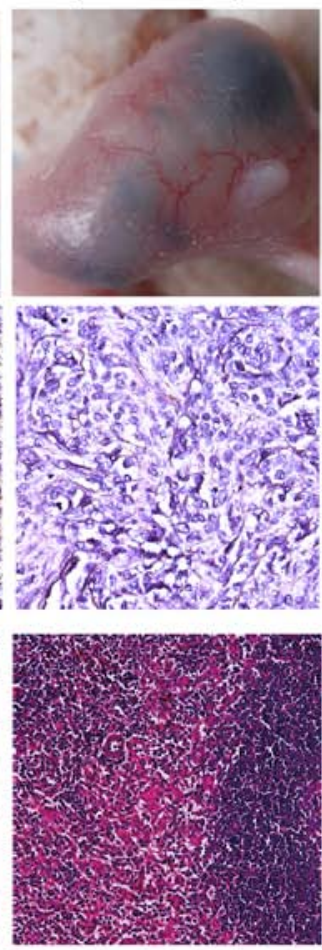

C

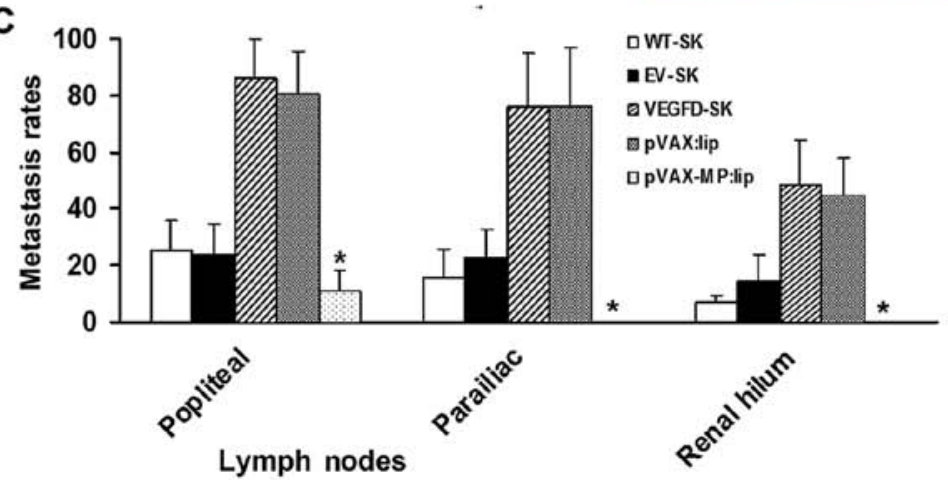

Figure 4. Qualitative and quantitative analyses of LVD and metastasis rate of LNs. (A) Top panel, qualitative LV was evaluated by Even's blue lymphangiogram. There are dilated LVs around tumors in VEGFD-SK and pVAX:lip groups (arrow, blue lymphatic vessels). Nearly no visible LVs were seen when VEGFD-SK tumors were treated with pVAX-MP:lip. A few of LVs appeared in the WT-SK and EV-SK groups. Middle panel, LYVE-1 staining shows that rich nascent LVs present in VEGFD-SK and pVAX:lip group. However, very few LVs appear in pVAX-MP:lip group. Sparse LVs in WT-SK and EV-SK groups were detected. Bottom panel, microscopic images show that clumpy deposits of metastatic growth have occupied almost entire the LNs tissues in VEGFD-SK and pVAX:lip groups, but very few metastasis was established in pVAX-MP:lip group. (B) Quantitative analyses of LVD were calculated by LYVE-1 staining. Tumor in VEGFD-SK and pVAX:lip groups displayed increased LVD compared with tumor in WT-SK and EV-SK groups. However, pVAX-MP:lip group show obviously reductions in LVD ( $\mathrm{P}<0.05$ versus WT-SK and EV-SK groups respectively). (C) The comparison of metastasis rates in tumor-draining LNs show that VEGFD-SK and pVAX:lip groups have a much higher metastasis tendency than other groups (arrow, tumor cells in lymph node). The VEGF-D-enhanced metastases to multilevel LNs were evidently reversed after the pVAX-MP:lip administration ( ${ }^{*} \mathrm{P}<0.05$ versus other groups).

of LVD compared with the LVD of WT-SK $(23.9 \pm 7.75)$ and EV-SK $(36.7 \pm 7.87)$ groups, whereas pVAX-MP:lip group showed a tendency of reduction of LVD (10.1 \pm 7.77$)$. Data of the microvessels with five high-power fields (HPF) are represented as the mean $\pm \mathrm{SD}$ (Fig. 4B) (" $\mathrm{P}<0.05$ versus any other group).

pVAX-MP:lip reversed high metastasis induced by VEGF-D. Histopathological assay showed that clumpy deposits of metastatic growth had occupied almost entire lymph nodes in VEGFD-SK and pVAX:lip groups (arrow, tumor cells in lymph node), but very few or no metastasis was established in pVAX-MP:lip group (Fig. 4A, bottom panel). The metastasis comparison for tumor-draining lymph nodes showed that
VEGFD-SK and pVAX:lip groups had a much higher metastasis tendency than other groups. The enhanced metastasis of multilevel lymph nodes induced by VEGF-D were evidently reversed after the pVAX-MP:lip administration, with zero metastasis in renal hilum LNs of pVAX-MP:lip group. Rate of metastasis comparisons were expressed as the number of metastatic lymph node counts out of the total lymph nodes of each barrier among the five groups, according to the three-step barrier LNs (Fig. 4C), namely, barrier one popliteal LNs (WT-SK, $25.2 \pm 10.7 \%$; EV-SK, $23.7 \pm 10.9 \%$; VEGFD-SK, $86.1 \pm 13.7 \%$; pVAX:lip, $80.28 \pm 15.29 \%$; pVAX-MP:lip, $7.5 \pm 10.7 \%$ ); barrier two parailiac LNs (WT-SK, 15.6 $\pm 10.1 \%$; EV-SK, $22.5 \pm 10.3 \%$; VEGFD-SK, $76.4 \pm 18.3 \%$; pVAX:lip, $75.83 \pm 21.3 \%$; pVAX- 


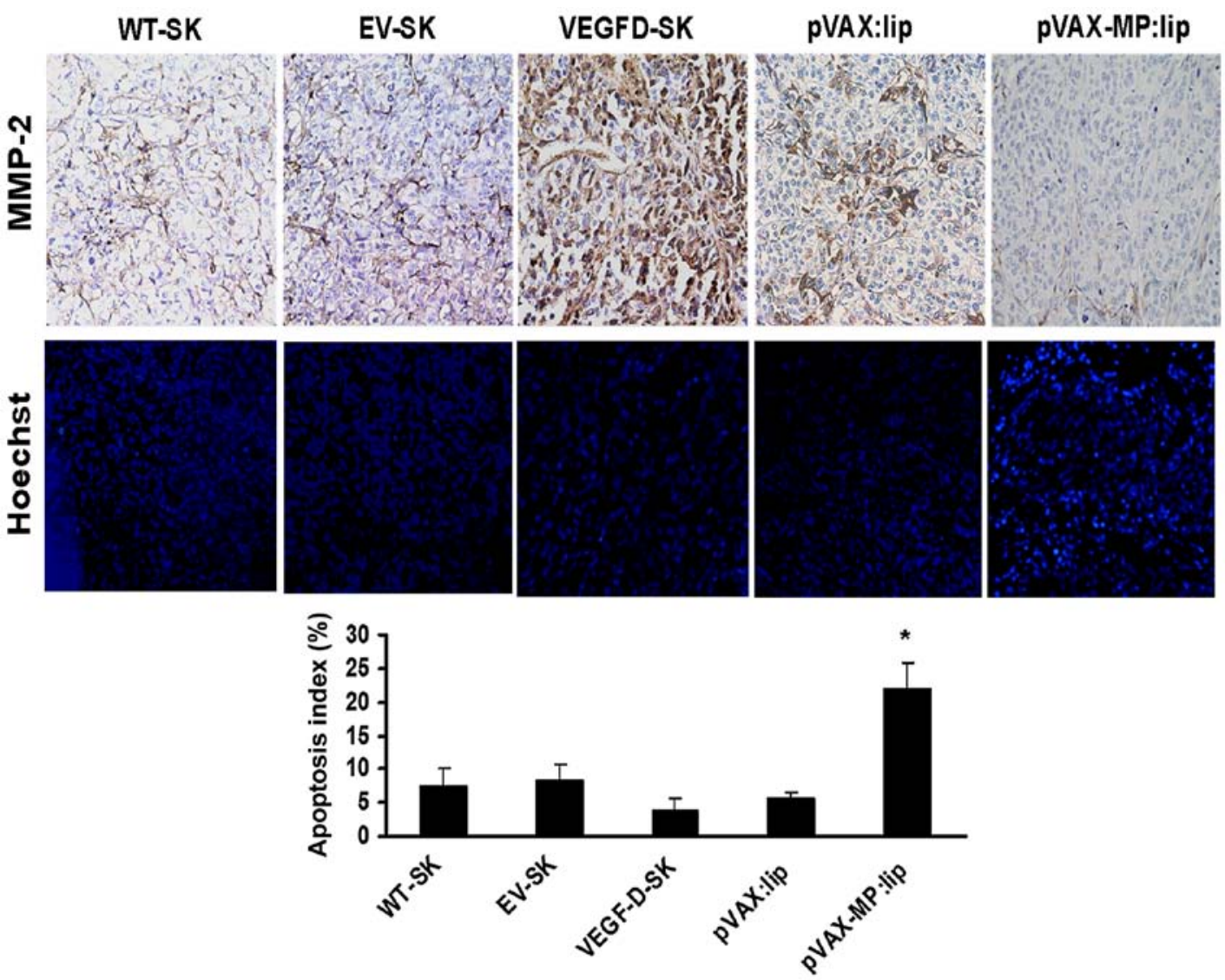

Figure 5. Expression of MMP-2 and comparisons of apoptotic indexes among different groups. Top panel, there was much stronger MMP-2 staining in tumor tissue of VEGFD-SK group compared with the weak staining in WT-SK group and EV-SK group. The pVAX:lip group also exhibited deep MMP-2 staining; whereas the pVAX-MP:lip group showed negative MMP-2 staining. Middle panel, microscopic fluorescence images show apoptotic cells of tumor tissues of different groups by Hoechst-33258 staining. VEGFD-SK tumor tissue manifested a few apoptosis of tumor cells; whereas pVAX-MP:lip group tumor tissue revealed a significant enhancement in apoptosis of tumor cells (arrow, apoptotic cell). However, no appreciable difference was observed between WT-SK group and EV-SK group. Bottom panel, VEGFD-SK group tumor tissue manifested a significant suppression of apoptotic tumor cells in contrast to other groups. Whereas higher apoptosis index was observed after the pVAX-MP:lip administration (" $\mathrm{P}<0.05$ versus other groups).

MP:lip, 0); and barier three renal hilum LNs (WT-SK, 6.7 $\pm 2.5 \%$;

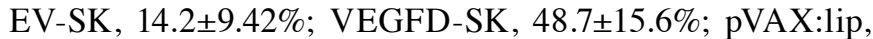
44.72 $\pm 13.14 \%$; pVAX-MP:lip, 0). VEGFD-SK group had much higher metastasis tendency in each barrier, but pVAX-MP:lip group had the lowest or zero metastasis tendency $(\mathrm{P}<0.05)$.

Expression of MMP-2. The microscopic column showed expression of MMP-2 of local tumor in different groups (Fig. 5, top panel). In comparison with the weak stromal staining (+) in WT-SK group and EV-SK group, $>90 \%$ tumor cells and stroma of VEGFD-SK group showed much stronger staining for MMP-2 (+++). The pVAX:lip group exhibited deep MMP-2 staining, especially in part of tumor cells $(++)$; whereas the pVAX-MP:lip group showed slight MMP-2 staining.

Apoptosis of tumor cells in vivo. Microscopic fluorescence images showed apoptotic cells of tumor tissues in different groups by Hoechst-33258 staining (Fig. 5, middle panel). Apoptosis index of VEGFD-SK tumors $(3.9 \pm 1.7)$ was much lower than that of any other group (Fig. 5, bottom panel). No appreciable differences were observed between WT-SK group (7.3 \pm 2.68$)$ and EV-SK group (8.28 \pm 2.31$)$. Lower apoptosis index was also observed in pVAX:lip group $(5.571 \pm 0.903)$. Whereas, higher apoptosis index was only detected after the

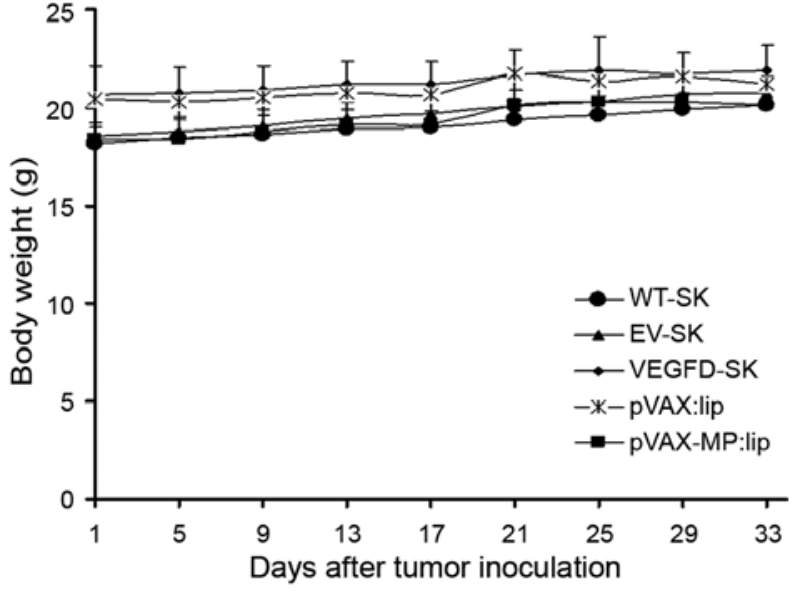

Figure 6. Mouse weight. No significant differences in mouse weight was found among the five groups $(\mathrm{P}>0.05)$.

pVAX-MP:lip administration $(21.875 \pm 4.01, \mathrm{P}<0.05$ versus any other group).

Safety. No significant differences in the weight of mice were found among the five groups ( $\mathrm{P}>0.05)$ (Fig. 6). No gross 
abnormalities were detected in this study. Additionally, toxic reaction was confirmed by $\mathrm{H} \& \mathrm{E}$ staining. The results indicated that there were no significant pathologic alterations in major organs among the five groups (data not shown).

\section{Discussion}

Ovarian cancer has the highest mortality rate of the malignancies of the female reproductive tract. Lymph node metastasis is a major prognostic factor in ovarian cancer. However, there is no feasible therapeutic strategy against lymphatic metastasis of ovarian cancer at present.

Metastasis to lymph nodes via lymphatic vessels is a common step in the dissemination of ovarian cancer. Although the molecular mechanisms of how lymphangiogenesis facilitates metastasis remains unclear, research has suggested that VEGF-D is associated with lymphangiogenesis-induced tumor metastasis to lymph node by stimulating LV proliferation and increasing the diameter and number of LVs (26). Lymphangiogenesis can exist in the tumor mass and proliferate rapidly in the presence of VEGF-D. With increasing numbers of LVs, as passage for tumor cells, the overexpression of VEGF-D further encourages lymphatic metastasis. It has been reported that high expression of VEGF-D results in accelerated tumor growth and develops metastasis via the lymphatic vessels $(9,10)$. In this study, we prepared a lymph node metastatic mouse model using SKOV3 transfected with recombinant pcDNA3.1/ VEGF-D plasmid. Strong positive expression of VEGF-D in SKOV3 tumor cells were detected both in vitro and in vivo. In addition, our study showed high expression of MMP-2 in agreement with the overexpression of VEGF-D, which might be associated with easy and high metastasis of tumor cells via LVs $(27,28)$.

At present, therapeutic intervention by neutralization of VEGF-D has become a promising approach for the treatment of malignant diseases (29). Recent studies have indicated soluble VEGFR-3 protein or monoclonal antibodies which could block the VEGF-D pathways have been used as a potentially biotherapeutic agent for inhibition of metastatic spread and collapse of tumor vessels $(9,30,31)$. High-level expression of VEGF-D could induce invasive and metastatic behaviors, while lymphangiogenesis and lymphatic metastasis were inhibited by knock-down of VEGF-D (32).

MP is capable of inducing apoptosis via inhibiting host gene expression (11-14), nucleocytoplasmic transport of host RNA and proteins $(15,16)$ as well as inactivation of Akt $(17)$.

It has been reported to possess potent antitumor and anti-angiogenesis properties (18-20). However, whether MP could play a suppressive role in lymphatic metastasis remains unclear. In this study, the antitumor and antimetastasis efficacy of a recombinant plasmid DNA carrying MP-cDNA in the lymphogenous metastatic model was tested. MP showed anti-lymphangiogenic and anti-lymphatic metastasic properties efficiently by analysis of lymphography, LVD, and pelvic lymph node metastasis rates in mice. VEGF-D-enhanced metastasis to multilevel lymph nodes in ovarian carcinoma were evidently reversed after administration with pVAX-MP:lip. It is especially worth noting that the nodal metastasis in pVAX-MP:lip group was evidently blockaded and there is zero metastasis in parailiac and renal hilum LNs. Moreover, MP exhibited potent antitumor efficiency, and resulted in more than $80 \%$ inhibition in tumor volume compared with empty vector.

Tumor progression and metastasis is a complex process involving multiple cellular steps and mechanisms. To elucidate the possible mechanism underlying the antitumor and antimetastatic property of MP, expression of VEGF-D was evaluated. Overexpression of VEGF-D was observed in the VEGFD-SK cells. However, the expression of the exogenous VEGF-D was decreased obviously when interfered with MP. Some evidence has demonstrated that lymphangiogenesis of sprout plays a significant role in cancer progression and prognosis (33-36). In this study, the high-LVD induced by VEGF-D was declined significantly after MP administration.

Lymphangiogenesis is the result of the synergy of many factors such as tumor and VEGF-D. MP not only reduces VEGF-D, but also induce tumor cell apoptosis. Therefore, in our study, the reduction of lymphangiogenesis is more obvious than the repression of VEGF-D with the impact of MP.

Stimulation of motility and invasiveness represent key steps in the process of metastasis by both vascular and lymphatic routes. In vitro approaches including migration and invasion assays for tumor cells revealed that anti-lymphatic metastasis role of MP in ovarian cancer might be related to the suppression of invasion and migration. Expression of MMP-2 is associated with invasive and metastatic behavior (37-39). MMP-2 has the ability to degrade type IV collagen in the basement membrane, and thus has an important influence in tumor progression. In this study, transfection of VEGF-D resulted in upregulation of MMP-2. However, inhibition of MMP-2 expression by MP was observed distinctly in VEGFD-SK ovarian cancer. A significant suppression of apoptotic tumor cells was observed in VEGFD-SK ovarian carcinoma, but MP could result in significant apoptotic tumor cells by apoptosis assays in vitro and in vivo. Our data are consistent with the previously reported mechanism that MP could induce cytopathogenesis $(18,19,23)$.

Tumor size has been regarded as a stimulating factor of nodal invasion (40-42). It may be partly attributed to high interstitial pressure as the results of few numbers of lymph vessels, and of the worse microenvironment for tumor metastasis resulting from inhibition of MMP-2 expression. We observed that tumor growth was stimulated by the increased expression of VEGF-D. The enhanced tumor growth was inhibited by MP therapy. Angiogenesis is necessary in tumor growth and metastasis because the blood vessels supply malignant cells with sufficient oxygen and nutrients. In the previous study, antiangiogenesis effect of MP has been confirmed. MP can inhibit formation of neovascularization and induce mouse pancreatic islet endothelial cell (MS1 cell line) apoptosis (18). Similar effect was observed in our research (data not show). It may be another reason for the low lymphatic metastasis rate in pVAXMP:lip group.

The reversal of metastasis might be due to: i) MP significantly suppressed the growth and metastasis potential of the local tumor; ii) MP significantly suppressed VEGF-D-induced lymphatic sprouting; iii) MP directly inhibited the growth of nodal metastasis. Further studies are still necessary to elucidate the precise mechanisms of anti-lymph node metastasis and antitumor effect of MP.

In conclusion, VEGF-D promoted tumor metastasis via lymph system in the mouse model. The overexpression of 
VEGF-D, VEGF-D-enhanced cell invasion, migration, lymph node metastasis and lymphangiogenesis of ovarian carcinoma could be reversed by MP. Inhibition of tumor growth and suppression of MMP-2 expression were detected. Our results may provide a potentially effective therapeutic strategy against human lymphatic metastasis of advanced ovarian cancer without systemic toxic effects.

\section{Acknowledgements}

We thank Dr Ping Chen for his expert technical assistance. Dr Xinjiang Xie provided recombined pcDNA3.1/VEGF-D plasmid. Steven Pan helped in polishing the English, and provided with other useful suggestions. This study was supported by National Key Basic Research Program of China (973) (Grant No. 2011CB910703).

\section{References}

1. Edwards BK, Brown ML, Wingo PA, Howe HL, Ward E, Ries LA, Schrag D, Jamison PM, Jemal A, Wu XC, et al: Annual report to the nation on the status of cancer, 1975-2002, featuring population-based trends in cancer treatment. J Natl Cancer Inst 97: 1407-1427, 2005.

2. Siegel R, Naishadham D and Jemal A: Cancer statistics, 2012. CA Cancer J Clin 62: 10-29, 2012.

3. Concin N, Hefler L, van Bavel J, Mueller-Holzner E, Zeimet A, Daxenbichler G, Speiser P, Hacker N and Marth C: Biological markers in pT1 and pT2 ovarian cancer with lymph node metastases. Gynecol Oncol 89: 9-15, 2003.

4. Rao G, Crispens M and Rothenberg ML: Intraperitoneal chemotherapy for ovarian cancer: Overview and perspective. J Clin Oncol 25: 2867-2872, 2007.

5. Ayhan A, Gultekin M, Taskiran C, Celik NY, Usubutun A, Kucukali $\mathrm{T}$ and Yuce K: Lymphatic metastasis in epithelial ovarian carcinoma with respect to clinicopathological variables. Gynecol Oncol 97: 400-404, 2005.

6. di Re F, Baiocchi G, Fontanelli R, Grosso G, Cobellis L, Raspagliesi F and di Re E: Systematic pelvic and paraaortic lymphadenectomy for advanced ovarian cancer: Prognostic significance of node metastases. Gynecol Oncol 62: 360-365, 1996.

7. Millauer B, Wizigmann-Voos S, Schnürch H, Martinez R, Møller NP, Risau W and Ullrich A: High affinity VEGF binding and developmental expression suggest Flk-1 as a major regulator of vasculogenesis and angiogenesis. Cell 72: 835-846, 1993.

8. Kaipainen A, Korhonen J, Mustonen T, van Hinsbergh VW, Fang GH, Dumont D, Breitman M and Alitalo K: Expression of the fms-like tyrosine kinase 4 gene becomes restricted to lymphatic endothelium during development. Proc Natl Acad Sci USA 92: 3566-3570, 1995.

9. Stacker SA, Caesar C, Baldwin ME, Thornton GE, Williams RA, Prevo R, Jackson DG, Nishikawa S, Kubo H and Achen MG: VEGF-D promotes the metastatic spread of tumor cells via the lymphatics. Nat Med 7: 186-191, 2001.

10. Wang J, Guo Y, Wang B, Bi J, Li K, Liang X, Chu H and Jiang H: Lymphatic microvessel density and vascular endothelial growth factor-C and -D as prognostic factors in breast cancer: A systematic review and meta-analysis of the literature. Mol Biol Rep 39: 11153-11165, 2012

11. Black BL and Lyles DS: Vesicular stomatitis virus matrix protein inhibits host cell-directed transcription of target genes in vivo. J Virol 66: 4058-4064, 1992.

12. Gerlier D and Lyles DS: Interplay between innate immunity and negative-strand RNA viruses: Towards a rational model. Microbiol Mol Biol Rev 75: 468-490, 2011.

13. Ahmed M and Lyles DS: Effect of vesicular stomatitis virus matrix protein on transcription directed by host RNA polymerases I, II, and III. J Virol 72: 8413-8419, 1998.

14. Yuan H, Puckett S and Lyles DS: Inhibition of host transcription by vesicular stomatitis virus involves a novel mechanism that is independent of phosphorylation of TATA-binding protein (TBP) or association of TBP with TBP-associated factor subunits. J Virol 75: 4453-4458, 2001.
15. Ahmed M, McKenzie MO, Puckett S, Hojnacki M, Poliquin L and Lyles DS: Ability of the matrix protein of vesicular stomatitis virus to suppress beta interferon gene expression is genetically correlated with the inhibition of host RNA and protein synthesis. J Virol 77: 4646-4657, 2003.

16. Petersen JM, Her LS, Varvel V, Lund E and Dahlberg JE: The matrix protein of vesicular stomatitis virus inhibits nucleocytoplasmic transport when it is in the nucleus and associated with nuclear pore complexes. Mol Cell Biol 20: 8590-8601, 2000.

17. Dunn EF and Connor JH: Dominant inhibition of Akt/protein kinase B signaling by the matrix protein of a negative-strand RNA virus. J Virol 85: 422-431, 2011.

18. Zhong Q, Wen YJ, Yang HS, Luo H, Fu AF, Yang F, Chen LJ, Chen X, Qi XR, Lin HG, et al: Efficient inhibition of cisplatinresistant human ovarian cancer growth and prolonged survival by gene transferred vesicular stomatitis virus matrix protein in nude mice. Ann Oncol 19: 1584-1591, 2008.

19. Gou M, Men K, Zhang J, Li Y, Song J, Luo S, Shi H, Wen Y, Guo G, Huang M, et al: Efficient inhibition of C-26 colon carcinoma by VSVMP gene delivered by biodegradable cationic nanogel derived from polyethyleneimine. ACS Nano 4: 5573-5584, 2010.

20. Ahmed M, Puckett S and Lyles DS: Susceptibility of breast cancer cells to an oncolytic matrix (M) protein mutant of vesicular stomatitis virus. Cancer Gene Ther 17: 883-892, 2010.

21. Zhou Y, Wen F, Zhang P, Tang R and Li Q: Matrix protein of vesicular stomatitis virus: A potent inhibitor of vascular endothelial growth factor and malignant ascites formation. Cancer Gene Ther 20: 178-185, 2013.

22. Wen J, Fu AF, Chen LJ, Xie XJ, Yang GL, Chen XC, Wang YS, Li J, Chen P, Tang MH, et al: Liposomal honokiol inhibits VEGFD-induced lymphangiogenesis and metastasis in xenograft tumor model. Int J Cancer 124: 2709-2718, 2009.

23. Lin X, Chen X, Wei Y, Zhao J, Fan L, Wen Y, Wu H and Zhao X: Efficient inhibition of intraperitoneal human ovarian cancer growth and prolonged survival by gene transfer of vesicular stomatitis virus matrix protein in nude mice. Gynecol Oncol 104: 540-546, 2007.

24. Chen X, Wang X, Wang Y, Yang L, Hu J, Xiao W, Fu A, Cai L, $\mathrm{Li} \mathrm{X}$ and $\mathrm{Ye} \mathrm{X}$ : Improved tumor-targeting drug delivery and therapeutic efficacy by cationic liposome modified with truncated bFGF peptide. J Control Release 145: 17-25, 2010.

25. Zhou L, Du L, Chen X, Li X, Li Z, Wen Y, Li Z, He X, Wei Y, Zhao X, et al: The antitumor and antimetastatic effects of N-trimethyl chitosan-encapsulated camptothecin on ovarian cancer with minimal side effects. Oncol Rep 24: 941-948, 2010.

26. Jussila L and Alitalo K: Vascular growth factors and lymphangiogenesis. Physiol Rev 82: 673-700, 2002.

27. Kabashima A, Maehara Y, Kakeji Y, Baba H, Koga T and Sugimachi K: Clinicopathological features and overexpression of matrix metalloproteinases in intramucosal gastric carcinoma with lymph node metastasis. Clin Cancer Res 6: 3581-3584, 2000.

28. Mitra A, Chakrabarti J, Banerji A and Chatterjee A: Cell membrane-associated MT1-MMP dependent activation of MMP-2 in SiHa (human cervical cancer) cells. J Environ Pathol Toxicol Oncol 25: 655-666, 2006.

29. Griffioen AW: Lymphangiogenesis factors: a target for therapy? Blood 113: 4135-4136, 2009.

30. Kubo H, Fujiwara T, Jussila L, Hashi H, Ogawa M, Shimizu K, Awane M, Sakai Y, Takabayashi A, Alitalo K, et al: Involvement of vascular endothelial growth factor receptor-3 in maintenance of integrity of endothelial cell lining during tumor angiogenesis. Blood 96: 546-553, 2000.

31. Pytowski B, Goldman J, Persaud K, Wu Y, Witte L, Hicklin DJ, Skobe M, Boardman KC and Swartz MA: Complete and specific inhibition of adult lymphatic regeneration by a novel VEGFR-3 neutralizing antibody. J Natl Cancer Inst 97: 14-21, 2005.

32. Majumder M, Tutunea-Fatan E, Xin X, Rodriguez-Torres M, Torres-Garcia J, Wiebe R, Timoshenko AV, Bhattacharjee RN, Chambers AF and Lala PK: Co-expression of $\alpha 9 \beta 1$ integrin and VEGF-D confers lymphatic metastatic ability to a human breast cancer cell line MDA-MB-468LN. PLoS One 7: e35094, 2012.

33. Renyi-Vamos F, Tovari J, Fillinger J, Timar J, Paku S, Kenessey I, Ostoros G, Agocs L, Soltesz I and Dome B: Lymphangiogenesis correlates with lymph node metastasis, prognosis, and angiogenic phenotype in human non-small cell lung cancer. Clin Cancer Res 11: 7344-7353, 2005.

34. Hall FT, Freeman JL, Asa SL, Jackson DG and Beasley NJ: Intratumoral lymphatics and lymph node metastases in papillary thyroid carcinoma. Arch Otolaryngol Head Neck Surg 129: 716-719, 2003 
35. Dadras SS, Paul T, Bertoncini J, Brown LF, Muzikansky A, Jackson DG, Ellwanger U, Garbe C, Mihm MC and Detmar M: Tumor lymphangiogenesis: A novel prognostic indicator for cutaneous melanoma metastasis and survival. Am J Pathol 162: 1951-1960, 2003.

36. Bono P, Wasenius VM, Heikkilä P, Lundin J, Jackson DG and Joensuu H: High LYVE-1-positive lymphatic vessel numbers are associated with poor outcome in breast cancer. Clin Cancer Res 10: 7144-7149, 2004

37. O'Grady A, Dunne C, O'Kelly P, Murphy GM, Leader M and Kay E: Differential expression of matrix metalloproteinase (MMP)-2, MMP-9 and tissue inhibitor of metalloproteinase (TIMP)-1 and TIMP-2 in non-melanoma skin cancer: Implications for tumour progression. Histopathology 51: 793-804, 2007.

38. Taniwaki K, Fukamachi H, Komori K, Ohtake Y, Nonaka T, Sakamoto T, Shiomi T, Okada Y, Itoh T, Itohara S, et al: Stromaderived matrix metalloproteinase (MMP)-2 promotes membrane type 1-MMP-dependent tumor growth in mice. Cancer Res 67: 4311-4319, 2007
39. Egeblad $\mathrm{M}$ and Werb Z: New functions for the matrix metalloproteinases in cancer progression. Nat Rev Cancer 2: 161-174, 2002.

40. Nathanson SD, Anaya P, Avery M, Hetzel FW, Sarantou T and Havstad S: Sentinel lymph node metastasis in experimental melanoma: Relationships among primary tumor size, lymphatic vessel diameter and $99 \mathrm{mTc}$-labeled human serum albumin clearance. Ann Surg Oncol 4: 161-168, 1997.

41. Carter CL, Allen C and Henson DE: Relation of tumor size, lymph node status, and survival in 24,740 breast cancer cases. Cancer 63: 181-187, 1989.

42. Nagamoto N, Saito Y, Ohta S, Sato M, Kanma K, Sagawa M, Takahashi S, Usuda K, Nakada T and Hashimoto K: Relationship of lymph node metastasis to primary tumor size and microscopic appearance of roentgenographically occult lung cancer. Am J Surg Pathol 13: 1009-1013, 1989. 